\title{
Development of a revised conceptual framework of physical training for measurement validation and other applications
}

Received: October 15, 2020

For correspondence: Annie.Jeffries@uts.edu.au

\author{
Jeffries $A C,{ }^{1}$ \\ Marcora $\mathrm{S}^{2,3}$ \\ Coutts AJ, ${ }^{1}$ \\ Wallace $\mathrm{L}^{1}$ \\ McCall $A^{1,4}$ \\ Impellizzeri FM, ${ }^{1}$
}

1, University of Technology Sydney (UTS), Human Performance Research Centre, Faculty of Health, Sydney, NSW, Australia

2, Endurance Research Group, School of Sport and Exercise Sciences, University of Kent, Canterbury, UK

3, Department of Biomedical and Neuromotor Sciences (DIBINEM), University of Bologna, Bologna, Italy

4, Arsenal Performance and Research Team, Arsenal Football Club, London, UK

Please cite as: Jeffries, A., Marcora, S., Coutts, A. J., Wallace, L., McCall, A., \& Impellizzeri, F. M. (2020, October 19). Development of a revised conceptual framework of physical training for measurement validation and other applications. https://doi.org/10.31236/osf.io/wpvek modified on October, 2020. 


\title{
SportR \iv
}

\begin{abstract}
A conceptual framework has a central role in the scientific process. Its purpose is to synthesize evidence, assist in understanding phenomena, inform future research and act as a reference operational guide in practical settings. We propose an updated conceptual framework intended to facilitate the validation and interpretation of physical training measures. This revised conceptual framework was constructed through a process of qualitative analysis involving a synthesis of the literature, analysis and integration with existing frameworks (Banister and PerPot models). We identified, expanded and integrated four concepts that are important in the conceptualization of the process and outcomes of physical training. These were: 1) formal introduction of a new measurable component 'training effects', a higher order construct resulting from the combined effect of four possible responses (acute and chronic, positive and negative); 2) explanation, clarification and examples of training effect measures such as functional (performance-based), physiological, subjective and other measures (cognitive, biomechanical, etc.); 3 ) integration of the sport performance outcome continuum (from performance improvements to overtraining); 4) extension and definition of the network of linkages (uni and bidirectional) between individual and contextual factors and other constructs. Additionally, we provided examples of theoretical and practical applications of the conceptual framework such as validation and conceptualization of constructs (e.g. performance readiness), and understanding of higher order constructs, such as training tolerance when monitoring training to adapt to individual responses and effects. This conceptual framework provides an overarching model that may help understand and guide the development, validation, implementation, and interpretation of measures used for athlete monitoring.
\end{abstract}

Keywords: Training response, contextual factors, performance, subjective measures, acute, chronic, athlete monitoring

\section{INTRODUCTION}

\section{Aims of the Conceptual Framework}

\subsection{What is a Conceptual Framework?}

A conceptual framework synthesizes evidence, assists in understanding the phenomena under investigation, informs future research and acts as a reference guide in practical settings. In the scientific process, conceptual frameworks allow more precise specification of hypotheses, thus increasing their degree of falsifiability, including when auxiliary or primary hypotheses of the main theory are modified if predictions fail $[1,2]$. This prevents ad hoc modifications of the theories, i.e. bias. A conceptual framework may aid in the explanation of particular phenomena providing the background knowledge, based on which the potential causal structure is formally presented as directed acyclic graphs for statistical examination (counterfactual framework) [3, 4]. This also allows the identification of confounders, moderators, mediators, colliders etc. $[5,6]$. The second purpose of a conceptual framework is to guide the validation of measurements [7]. Lack of conceptualization and articulation of causal phenomena weakens research in a number of ways including poor methodological arguments, lack of conceptual clarity and undermining of the foundation of solid empirical research [8].
In the context of patient-reported outcome measures, a conceptual framework is a representation of the relationships between the construct to be measured (e.g. using reflective or formative models) [9]. This explanation is consistent with a generic definition, which describes a framework as the structure which the researcher believes to best explain the phenomenon to be studied [10]. It may take the form of a visual representation, arranged in a logical structure, illustrating presumed relationships between key concepts, constructs or variables. The availability of a reference conceptual framework, together with operational definitions of the constructs, is therefore essential for the measurement validation process [11]. This provides a basis for researchers to propose exactly what and why specific variables (objective or subjective) are measured and act as a reference for validation studies. Of particular importance is understanding "why" since a measure can be valid for a specific context and goal but not in another.

\subsection{Why Develop a Conceptual Framework of Physical Training?}

In the last decade, there has been an increased interest in subjective measures within sports science, with new constructs (e.g. wellness, wellbeing, performance readiness etc.) introduced but never clarified [12]. Moreover, while the use and development of reference theoretical and 
conceptual frameworks is common in disciplines such as psychometrics and clinimetrics [11, 13], they are not commonly used when validating measurements used in sport science. Unfortunately, the absence of a reference framework presenting hypothesized relations between these constructs and other measures (e.g. training load), makes the examination of the validity and usefulness of these items introduced in the sport science literature confusing and prone to bias (e.g. post hoc "theories" explaining relations, that are not subsequently tested and that decrease the degree of falsifiability) [14]. For example, some common subjective measures used in research and practice such as "wellness items" have never been validated following appropriate and established methods such as those presented by the COnsensus-based Standards for the selection of health Measurement Instruments (COSMIN) [12]. This may also be due to the fact that available frameworks [15, 16] do not include all components necessary to provide a suitable reference for these kinds of validation studies. For this reason, we re-examined available frameworks and proposed a conceptual framework that can facilitate the validation of physical training measures. The current conceptual framework refers to physical training, that is the training involving physical activities with the goal of improving sport performance. Physical training encompasses both process and outcomes. Training process is the systematic repetition of physical exercises involving external and internal load [17], and its outcome may include physiological, biochemical, anatomical, and functional changes i.e. training effects and sport performance outcomes [15]. The conceptual framework excludes other kinds of training such as tactical training, watching videos and pure psychological skills training that can improve sport performance but do not require physical exertion.

\section{Development Process of the Conceptual Framework}

The development of our conceptual framework (Figure 1) was constructed through a process of qualitative analysis. This was an integrative and evolving progression, consisting of a synthesis of the literature and analysis of existing frameworks, coupled with an assimilation of information and concepts from previously developed models and theories. We developed the conceptual framework for the training process using conceptualization, a process in which imprecise constructs and their constituent components are defined in precise terms [18]. The version presented in the current paper is the final result of more than 15 versions and elaborations, that were conceptually tested using a red team approach [19], where the authors challenged conceptually the framework by proposing (worst case) scenarios to test whether it could reasonably fit common measures and training strategies. By conceptualizing and adding other constructs (individual and contextual factors, and training process), we also defined their relationships within the conceptual framework. Below we have documented the main stages in the development process in which we explicitly justify and elucidate decisions about key elements of the conceptual framework.

\subsection{The Previous Version of the Physical Training Framework}

We decided to re-evaluate and refine a previous physical training framework [15, 16] integrating or modifying, together with the examination of other frameworks or models available in the literature. This original framework was developed to define and conceptualize the essential measurable components of the training process and its outcomes. Briefly, a fundamental notion of this physical training framework was that when delivered appropriately, and according to the training goal and plan, exercise will induce psychophysiological responses leading to adaptations. These responses during the exercise are the stimuli initiating the adaptations determining the training outcomes. The training load construct was differentiated into two components, external and internal training load, by redefining and elaborating two terms (external and internal) that were already present, but not operationally conceptualized, in some non-peer-reviewed coaching literature [20] and seminal textbooks on training periodization [21]. In this previous framework, the external training load, and its interaction with various individual and contextual factors, determines the internal training load, which ultimately will produce changes in the training outcome. For the operational definitions see Table 1 and for more details we refer the reader to a recent publication [16]. This previous theoretical framework has since been also adapted to other fields such as physical activity for health or other contexts such as biomechanics $[22,23]$.

\subsection{Identification of Other Frameworks}

A first attempt to systematically search the literature failed because, in sport science, frameworks for physical training are not presented as such and/or they are not clearly indicated in titles and abstracts. We therefore decided to locate suitable frameworks based on the expert knowledge of the authors, scoping searches and publication references. This may have introduced bias, although this is inevitable when developing conceptual frameworks given the qualitative nature of the process. From the examination of the literature, we identified and selected two other potentially relevant models specifically proposed for the training process: the Banister Impulse-Response (IR) [24, 25] and the Performance Potential Metamodel (PerPot) [26, 27]. The model of Banister considered athletic performance to be measurable as the net outcome of two key training responses, also called fitness and fatigue, from the 
application of a training impulse. The original works by Banister et al. [24, 25] applied a systems theory to evaluate the response to physical training using a mathematical function. In this mathematical model, performance is the output, with the athlete regarded as the system, and the training impulse as the input $[24,25]$. The functional relationship between the training impulse and the system response is expressed by two differential first order equations attributed to the antagonist effects that were called fitness and fatigue [24, 25]. The PerPot metamodel $[26,27]$, simulates the interaction between load and performance in adaptive physiological processes in sport, by means of antagonist dynamics (response flow and strain flow). The components of the PerPot $[26,27]$ have some similar theoretical characteristics to Banister et al, [24, 25] such as the concept of antagonist role of strain and response potential, whilst using a different computational approach. Finally, we also referred to the joint consensus statement of the European College of Sport Science (ECSS) and the American College of Sports Medicine (ACSM) on overtraining [28]. Although it was not formally presented as such, it can be seen as a theoretical framework presenting the continuum of the effects of training and individual/contextual factors on sport performance.

\subsection{Integration with Other Frameworks}

While these two models [24-27] included, albeit with different names, a negative and positive component, the theoretical framework of physical training by Impellizzeri et al. $[15,16]$ did not include these two elements. Therefore, we initially attempted to combine the two components (fitness and fatigue) of the IR-Banister model into a new framework. However, there was a conceptual problem related to the definitions, or lack thereof, for the terms 'fitness' and 'fatigue'. Originally, these two terms were arbitrarily used to define two mathematical components reflecting the positive and negative training responses. They were computationally and not conceptually defined since they just represented an assigned name for two "model components of performance ability" and not two theoretical constructs. However, the interpretation of these two terms may result in confusing overlapping with the more generic definitions reported in the literature, making it challenging to achieve a clear understanding of these constructs for conceptualizing into a general framework. For example, the ACSM defines fitness as "a set of attributes that people have or achieve that relates to the ability to perform physical activity" [29]. Fitness has been also operationalized as "[a set of] measurable health and skill-related attributes" that include cardio-respiratory fitness, muscular strength and endurance, body composition, flexibility, balance, agility, reaction time and power [30]. These definitions refer, however, to a set of attributes which are outcome measures (output) and do not reflect the fitness component of the IR
Banister model (input). Similarly, this problem also relates to the many definitions of fatigue [31-33], none reflecting the second input variable of the Banister model and even the strain of the PerPot. Indeed, fatigue is a complex construct that can be interpreted in terms of an acute reduction in muscle force/power, higher perception of effort, task failure, a mood dimension or as a symptom of disease depending on the context $[34,35]$. Whilst some kinds of fatigue can be considered negative effects of training, the term fatigue cannot be used as a synonym of negative effects because fatigue is not the only negative effect of training. For example, training can induce muscle damage and delayed-onset muscle soreness (DOMS) [36] and other negative alterations in mood such as an increase in depression [35]. Therefore, whilst we considered it worth adding these two competing components, we decided not to use the terms fitness and fatigue due to the above issues. We then replaced "fitness" and "fatigue" in Banister's model, with positive and negative training effects. This was still consistent with the original meaning of these two components that were defined as positive or negative based on their direct effects on performance [24, 37]. Additionally, it was also compatible with the PerPot model where Perl described the antagonist dynamics as: "the same load input has two contradictory effects, namely the performance increasing response flow [our positive training effects] and the performance decreasing strain flow [our negative training effects]" [27]. However, we did not want to use the term strain and response, as in the PerPot model, since these terms are loaded and may create confusion in the physical training context. We also specify that the PerPot model can be applied to various "performances" and not necessarily competitive performance. In the PerPot model, performance is a generic term that can include biological and physiological outcomes (e.g. hemoglobin and heart rate) $[26,27,38]$.

In a further evolution of our conceptual framework we expanded the training effect construct with the additional acute and chronic dimensions. Below, each section of the conceptual framework is described in further detail. Finally, we integrated the ECSS/ACSM [28] overtraining framework to define the possible sport performance outcomes resulting from the balance between positive and negative training effects.

\subsection{New and Expanded Concepts}

Above we identified the lack of the two positive and negative training effects in the previous framework $[15,16]$. We therefore adapted the previous framework to overcome this limitation and to integrate, elaborate and clarify concepts of existing frameworks. Specifically, we identified four concepts that could be integrated or extended. An 
iterative process was utilized to determine the final version of the conceptual framework.

The first concept we expand and integrate is the training effect, which in the previous framework $[15,16]$ was mixed with the generic concepts of 'adaptations' and of training outcome. However, it did not constitute a formal and defined construct of the framework. We acknowledge that the lack of the training effect construct may have contributed to confusion, where measures of effects occurring after the training session have been misinterpreted as measures of internal training load [39], which is by definition the psychobiological response during the exercise(s) constituting the training session [16].

The second aspect we more formally clarified is training effect measures. The training effect is a construct for which there is no gold standard measure however it can be assessed in several ways, including using proxy measures when the training effect being considered cannot be directly quantified. This is an important clarification since the validation process may also refer to proxy measures, e.g. whether measures are adequate reflections of the construct of interest.

The third area we further articulate is sport performance outcomes. In the previous frameworks of Impellizzeri, Rampinini [15] and Impellizzeri, Marcora [16] used the term 'training outcomes' which does not properly differentiate between the chronic training effect and sport performance outcomes. In the current conceptual framework, these two constructs are differentiated and sport performance outcomes further elaborated by including the potential range, i.e. from improvement to overtraining.

The fourth area we expand on is the concept of individual and contextual factors (e.g. training status, health, nutrition and environmental factors). In the previous frameworks, $[15,16]$ contextual factors were only mentioned as aspects influencing internal load and thereby training outcomes. Here, we clarify that modifiable and non-modifiable individual/contextual factors have a more widely integrated relationship with all components of our conceptual framework, including bidirectionality with training effects. These four areas are further discussed in the next section.

\section{Conceptual Framework}

This conceptual framework for physical training is intended to illustrate the relationship between stimulus (internal training load), training effects and their measures and sport performance. Similar to the previous versions, we wanted to develop a parsimonious conceptual framework presenting the essential measurable components such that it may be elaborated and expanded for specific applications and needs. The internal and external training load constructs have been presented in earlier sections and in previous publications $[15,16]$ and although essential parts of the current conceptual framework, will not be further discussed here. For the measurement of external and internal load we direct readers to the following reference [40]. We now provide details of the additional or modified constructs: training effects, sport performance outcomes, and individual/contextual factors. The conceptual framework (constructs and their relationships) is visually depicted in Figure 1. Operational definitions of each construct of the conceptual framework are presented in Table 1.

\subsection{Training Effects}

The main new construct added to this conceptual framework is the construct "training effect", which is the combination of four possible effects denoted by four quadrants (Figure 1). These four quadrants represent the possible acute/chronic and positive/negative effects that can influence sport performance and interact with each other, i.e. all permutations. We decided to operationally define acute and chronic training effects according to two related criteria: the amount of training required to induce a measurable effect and the time needed to return to baseline once training is stopped. Clearly, it is not possible to dichotomise the criteria since they should be seen as a continuum. However, from an operational point of view, we suggest that a training effect occurs after a single training session or few training sessions (up to one week of training) to be classified as acute, whilst the training effects occurring after more than one week of training as chronic. A similar time frame can be used with regards to return to baseline: training effects requiring less than one week to disappear should be classified as acute, whilst those lasting more than one week as chronic. Importantly, these are approximate criteria, and acute and chronic should be seen more as a continuum than a strict dichotomy.

The training effect can be both positive or negative and, as indicated by the conceptual framework, can occur concurrently. In other words, a positive effect (increased muscle protein synthesis after resistance training) [41] may be accompanied by a negative effect (neuromuscular fatigue) [42]. With the terms positive and negative, we indicate an acute or chronic effect that directly improves (positive) or directly impairs (negative) the sport performance outcome. This definition is consistent with the original Banister (and PerPot) model, where effects referred directly to performance [24-27]. More specifically, however, our use of the terms negative and positive qualifies the direct effects but does not necessarily imply that their indirect effects are also positive or negative. For example, glycogen depletion has a direct negative effect on endurance performance [43]. However, training in a glycogen depleted state can enhance activation of key cell signaling kinase 
transcription factors and transcriptional co-activators, resulting in a coordinated up-regulation of nuclear and mitochondrial genomes [44, 45] (indirect positive effect). Therefore, whilst the direct effect of glycogen depletion is negative, its indirect effect via enhanced mitochondrial biogenesis can have a positive effect on endurance performance $[44,45]$.

As mentioned previously, we have introduced acute and negative training effects to conceptually integrate the two negative and positive effects were computationally determined, but they both fit into the current conceptual framework, further supporting the plausibility of our proposal.

\section{Measures of training effects}

The components included in the training framework are constructs for which, commonly, there are no gold standards, but there are various measures, including

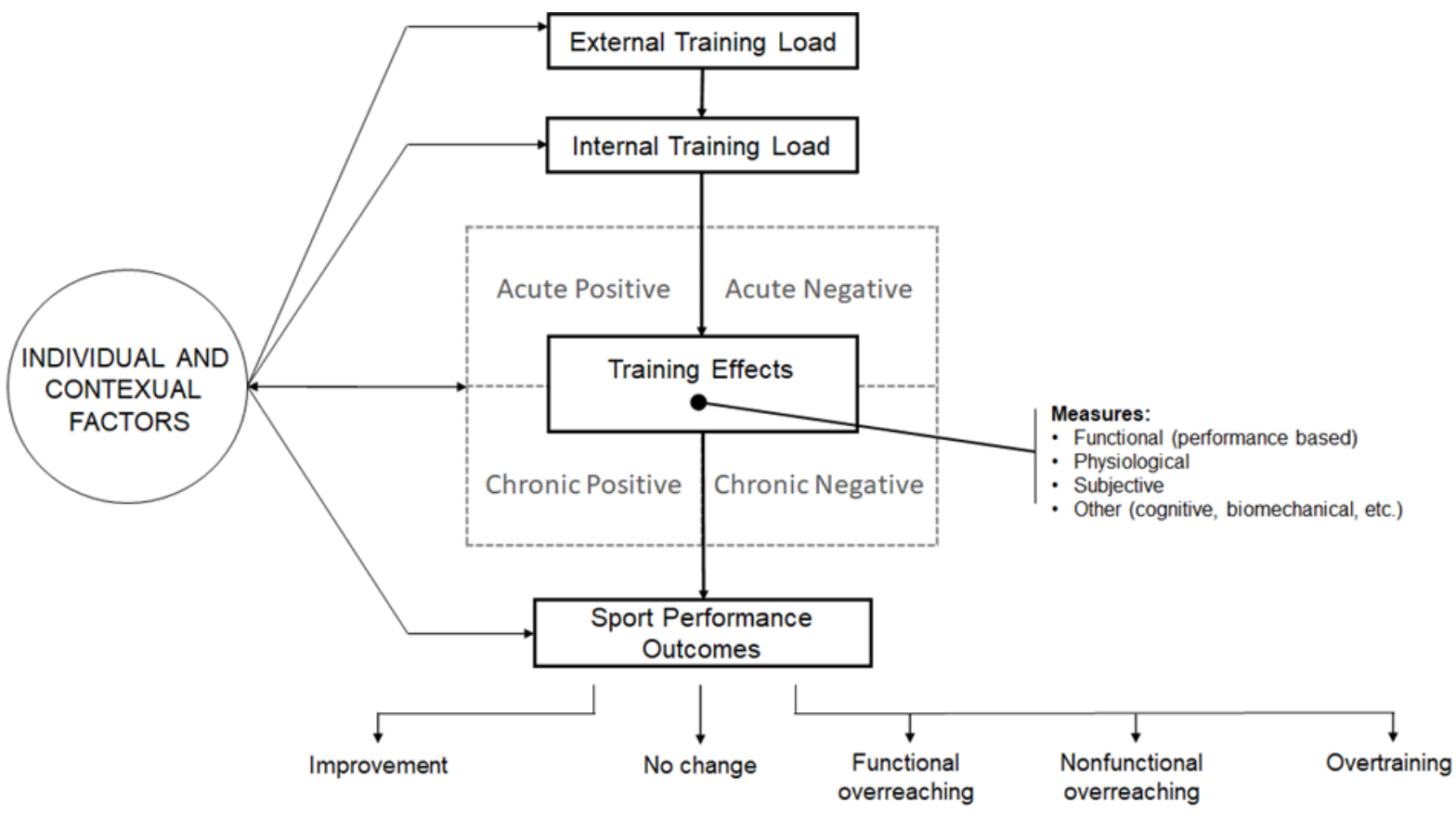

Figure 1. Conceptual framework

competing components from previous models (fatigue and fitness in the IR Banister model and strain and response flow in the PerPot model) [24-27]. However, a fundamental difference with these models is that while we integrated the concepts of two competing effects, we did not identify these effects with the input measures as in their models, where inputs are measures of external load. Indeed, we provided a conceptual framework, while the aforementioned models aimed to predict performance outcomes using computational methods.

Nevertheless, the current conceptual framework is coherent with these two models since they tried to predict performance outcomes by using external training load measures as proxies (surrogates) to quantify the negative and positive effects, with the balance between these two competing effects determining the performance. The use of these proxy measures is conceptually (and tautologically) supported by the causal relation between training effects and training load. The two models differed in the way the surrogate (proxy) measures reflecting the construct. New candidate measures can be proposed but they necessitate proper validation before implementing in practice and research, and the current framework provides the conceptual model to develop testable hypotheses to support their validity. Here we present and discuss some examples of training effect measures that we have classified, for simplicity, in four main categories as presented in Figure 1: 1) functional measures (performance-based), 2) physiological measures, 3) subjective measures, 4) other measures (cognitive, biomechanical, etc.).

\subsubsection{Functional Measures}

Functional measures are measures of performance during a task that is either related to competitive performance or that is thought to measure a specific fitness component (e.g. strength). An example of such measures commonly used in sport is the countermovement jump (CMJ) test. As many 
other measures of training effects, the $\mathrm{CMJ}$ test can be used in two ways. Firstly, to quantify neuromuscular fatigue [46, 47] which is an acute negative effect of training. In this case, the CMJ test is performed after the training session and a reduction compared to the values in rested conditions is a measure of neuromuscular fatigue $[48,49]$. The second use of the $\mathrm{CMJ}$ test is to measure the chronic effect of training on lower body power (proxy measure) or jumping performance. For this purpose, conditions need to be standardized to limit as much as possible the influence of acute training effects and contextual/individual factors. For example, by reducing the training load and allowing recovery from neuromuscular fatigue and by controlling time of testing and sleep, nutrition, motivation before and/or during test [50]. Standardization has the dual roles of increasing reliability [9] and providing a measure reflective of a chronic training effect [50]. Other examples of functional measures that are used to assess acute or chronic training effects are agility tests, sprints and multistage fitness tests [51-54].

\subsubsection{Physiological Measures}

There are various physiological measures that may be suitable for monitoring acute or chronic training effects. The better validated and most widely used are the physiological measures used to assess the positive chronic training effects of endurance training. These measures include maximal oxygen consumption, running economy and lactate threshold [55-57]. Although critical power or critical velocity are often considered measures of a physiological construct [55], we have classified as functional measures as they are based on performance during time to exhaustion tests or time trials (i.e. functional measures representing the observable outcomes of a physiological construct).

Another variable often monitored in athletes is creatine kinase (CK) because the elevated CK levels that may occur after eccentric and/or unaccustomed exercise are used as a proxy measure for muscle damage which is an acute negative training effect [58-60]. Another example of physiological measures are the assessment of central and peripheral fatigue using electrical or magnetic stimulation. However, contrary to the $\mathrm{CMJ}$ test mentioned earlier, these tests are less frequently utilized in practical settings due to the expertise required and need for specialized equipment $[61,62]$.

A physiological measure that can be used to measure both acute or chronic training effects is heart rate variability (HRV). Indeed, HRV can indicate acute post-exercise perturbation and recovery of the cardiac autonomic system (acute training effect). Additionally, HRV can signify chronic adaptations to training by means of vagally mediated indices (chronic training effect) $[63,64]$.

\subsubsection{Subjective Measures}

Subjective measures are commonly used for monitoring the training process. However, whilst the use of subjective measures is considered sound [65], this area is characterized by the widespread use in sport research and practice of improperly validated instruments [12]. Among the most utilized in research and practice (also implemented in athlete management systems and commercial software), are the so-called "wellness" items [12]. However, it is difficult to understand why the term "wellness" is used, given the lack of clarity surrounding this construct, and why it would be relevant in the training process. For example, is it intended to measure training effects (acute or chronic) or individual/contextual factors? The wellness items, depending on the versions, are supposed to measure fatigue, sleep quality, stress, muscle soreness, enjoyment of training, irritability, health causes of stress and unhappiness $[66,67]$. Even ignoring that these items have not been properly validated, it is not clear whether these items are supposed to measure acute effects, chronic or individual and contextual factors; that can be influenced by the acute training effects. Nevertheless, among the items, fatigue and muscle soreness are good candidates as measures of acute training effects. Constructs such as stress, irritability and unhappiness appear more as individual/contextual factors or chronic effects. However, this is speculative as no framework has been previously provided or used as reference. Therefore, we are using a "reverse engineering" approach, starting with the measures used and going back to understand what components of the training process they are supposed to measure. As such, it is difficult to suggest practical subjective measures of acute training effects other than muscle soreness, which is a validated measure, and fatigue for which validated single item instruments do not exist (at least in athletic populations), however multiple items instruments are available [68]. However, this highlights that a reference conceptual framework for validating these measures is needed (and one of the reasons why we developed it).

\subsubsection{Other Measures}

There are other potential measures that can be used to assess acute or chronic training effects. These include biomechanical (e.g. force-velocity profile, acceleration load) [69] or other more area specific measures such as cognitive tests $[70,71]$. For instance, assessment of acute training effects has been examined using cognitive function tests, such as the Stroop and response time tests, in elite cyclists and ultramarathon runners $[70,71]$. We have provided examples of measures that can be relatively easily implemented in practice (e.g. jump tests, CK, HRV). However, there are other measures that theoretically can be used but because of their complexity are relegated to the 
research area such as mitochondrial biogenesis, muscle protein synthesis and markers of various signal transduction pathways [72]. Whilst these cannot be measured in a practical manner (e.g. in a field setting), it does not mean they should be disregarded, as they play an integral role within the physical training process. Frameworks can be used to organize and contextualize fundamental science when thinking about training and its possible effects.

\subsection{Sport Performance Outcomes}

Sport performance outcomes are the result of the balance between positive and negative training effects, again consistent with the IR-Banister and PerPot models [24-27]. We provide a possible range of sport performance outcomes according to the ECSS/ACSM consensus on overtraining [28]. These include improvement, no change, and then a progression from functional to non-functional overreaching and finally over training [28]. Functional overreaching is used to improve performance and often applied during normal training cycles. It involves intensified training, which generally results in reduced performance, however after adequate rest an athlete's performance will improve relative to baseline levels. Conversely, if intensified training continues, without adequate rest, this may result in nonfunctional overreaching causing decrease in performance or stagnation potentially lasting for several weeks or more [28]. Despite this, with appropriate rest an athlete should fully recover. Towards the end of the continuum is overtraining in which performance decrement may last for months.

\subsection{Individual and Contextual Factors}

Contextual factors can be defined as all the factors not part of the main process (training) such as environmental, social, cultural factors, etc. [73]. Individual factors are characteristics of the individual athlete such as genetics, psychological traits and states, training background, etc. These factors interact, influence, alter, facilitate or constrain all the components of the training process [73]. In the conceptual framework, paths are depicted graphically by uni or bidirectional arrows. We conceptualized arrows as representing lines or directions of influence (potential causality). The bidirectional arrow represents a reciprocal nature of interactions between variables. We have included a bidirectional arrow between individual and contextual factors and the training effects. For example, a negative training effect (e.g. increased fatigue) can act as an individual factor subsequently influencing the internal training load in the following training session (indicated by the unidirectional arrow). On the other hand, other individual or contextual factors may also influence the training response (e.g. causing higher or lower negative effects) [74]. The unidirectional arrows indicate that the internal training load resulting from a specific external load will vary based on individual or contextual factors. For example, individual or contextual factors can moderate the effect of residual fatigue on the internal training load elicited by the planned external load. Similarly, modifications of individual and contextual factors can influence sport performance outcomes. For example, psychological status, nutrition (interventions or cultural aspects) or recovery strategies may impact sport performance outcomes, in either a negative or positive way [75-78]. Hypoxia induced by altitude training is an example of a contextual factor that can influence all the training process components, and that can be influenced by other contextual factors and interventions [79, 80]. Clearly, some individual and contextual factors are modifiable (health, nutritional status, environmental conditions, etc.) and others non modifiable (e.g. genetics, training history, etc.). We clarify that forms of training other than physical may be classified as contextual factors, that is strategies altering individual factors that can influence performance (e.g. mental or cognitive training) $[81,82]$.

\section{Examples of Practical and Theoretical Applications of the Conceptual Framework}

\subsection{Training Monitoring}

One practical utility of this conceptual framework is its application to training monitoring, that is the identification of the measurable components and their role within the training process. The conceptual framework together with the operational definitions allows us to understand what and why to measure these components, thus also facilitating the interpretation of the measures. As another practical example of application, it can be used to adjust and adapt the training process and periodization plans. Typically, periodization for athletic preparation focuses on the exercise component, by developing a program using external load, eventually estimating the induced internal training load using background knowledge and available evidence. In parallel, the planned external training load can be adjusted based on athlete responses and feedback [83]. This is an approach that may also require coaches to use heuristic methods [84]. Our conceptual framework does not present any innovative strategy in this regard [85], however it may assist in accommodating training based on individual responses by taking advantage of the measures that are nowadays possible to collect. We provide a model according to which the measures (type and timing) are selected based on what construct we want to quantify and for what reason (to measure an acute or chronic effect, an individual factor, etc.).

Overall, the measures of the component of the training process can assist in identifying and developing measures useful for informing decisions, controlling training load and 
determining if responses are progressing as intended, and/or the program needs to be modified. For example, a negative effect that is planned is not necessarily a problem. However, when a negative but unplanned effect occurs, this may be a signal of something amiss or something is changing (e.g. in contextual and individual factors). Therefore, modulation of external training load using feedback from the training effects, which are influenced by individual and contextual factors (bidirectional), may provide a supplementary "optimization" of the training process. With the term "optimization" we mean a training process which is flexible enough to be modified based on individual training responses and effects.

Differentiation between acute and chronic effects is also possible. The monitoring of acute training effects over time (time series) allows for the evaluation of trends to see if an acute effect is becoming chronic (e.g. fatigue). By adding a recovery short period (e.g. a few days, one week) may help identify if this trend indicates a chronic condition or whether this is just an acute and transient training effect (i.e. according to one of the criteria we proposed for the differentiation between acute and chronic). Other strategies to assess acute and not chronic negative effects can be to measure fatigue before and after training, with the difference used as acute fatigue induced by the training. The measure before training can be seen as an integration of an acute-chronic effect with individual and contextual factors. For example, fatigue due to recent and accumulated negative effects (chronic) combined with other factors such as nutrition and psychologically stressful situations outside of training. The difference between pre- and post-training measures of fatigue is the acute training effect.

\subsection{Training Tolerance}

It is common to refer in the training context to the ability of an athlete to cope with the demands of the training load. For example, to understand whether or how to adjust the training program, i.e. proceeding as planned or needing modification. The "ability to cope" may be defined as training tolerance, which is a "field" term commonly used and can be interpreted as a higher order construct, difficult to directly quantify. The proposed conceptual framework provides the opportunity and method to better define and assess training tolerance. For example, higher-than-normal fatigue (quantified using subjective or performance-based measures) caused by a typical internal training load may indicate lowering in training tolerance. Also, for consideration is the external training load, where for example, high levels of muscle soreness are to be expected when training includes eccentric exercise or in unaccustomed athletes $[58,86]$. However, if high levels of muscle soreness are reported after training, including primarily concentric exercise in accustomed athletes, this acute negative training effect may be indicative of poor training tolerance.

\subsection{Performance Readiness}

As mentioned in previous sections, subjective instruments may be used to assess training effects. Unfortunately, commonly used subjective measures have not been properly validated and as a consequence it is unclear whether they are actually measuring what they are supposed to measure, and what their role is within the training process. However, using a reference framework assists in understanding how, why and what constructs we wish to measure to support and understand the training process. Not without reason, the COSMIN guidelines require a reference framework for evaluating the validity of an instrument.

Understanding of the necessity and application of frameworks in the validation process is a conceptual matter grounded in research methodology. To explain how a framework can be used in the validation process, we provide an example of performance readiness, a concept widely used in sport settings, although never properly or clearly conceptualized [87]. The lack of conceptualization is evident from the measures used to assess this construct, and variations of the descriptors (e.g. player readiness, readiness to train). Furthermore, the same items for measuring wellness are also used for assessing performance readiness $[88,89]$. It is questionable how it is possible that the same items can measure two constructs. In an effort to provide an explanation, we speculate they implicitly represent two different but related constructs. Differentiating between these would require separate operational definitions and/or concept elicitation studies. Unfortunately, no such information or research exists. For exemplificative purposes, we refer to "wellness" to identify items commonly used in the sport science literature under this name [12]. The link between the two constructs can be hypothesized using the conceptual framework. Wellness items may reflect training effects, with performance readiness as a higher order construct influenced by or "incorporating" these subjective training effects. In one of the few attempts to provide an operational definition, Ryan et al. [90] proposed that optimal player readiness is a condition where an athlete has no impairment of physical performance, no mental fatigue or excessive psychological distress, and "the athlete's capacity to complete training activities and perform during competition". This is consistent with the use of this construct in the literature that refers to subsequent performance or physical training [91, 92]. Using this definition (others may be possible), physical performance seems to be the criterion for validation. Therefore, the lack of association between candidate training response effects, assumed to be related to performance readiness and hence 
subsequent training or competition physical performance, would not support the validity of these items as measures of performance readiness [91]. However, they still may be valid measures of training effects. For example, fatigue perception can be a valid measure of acute training effects, although may not be related to subsequent physical performance (e.g. high fatigue corresponding to lower external load) and therefore cannot be used for assessing performance readiness, or readiness to train.

To further illustrate, below we provide hypothetical examples for items measuring subjective training effects (e.g. wellness items) that may be related to performance

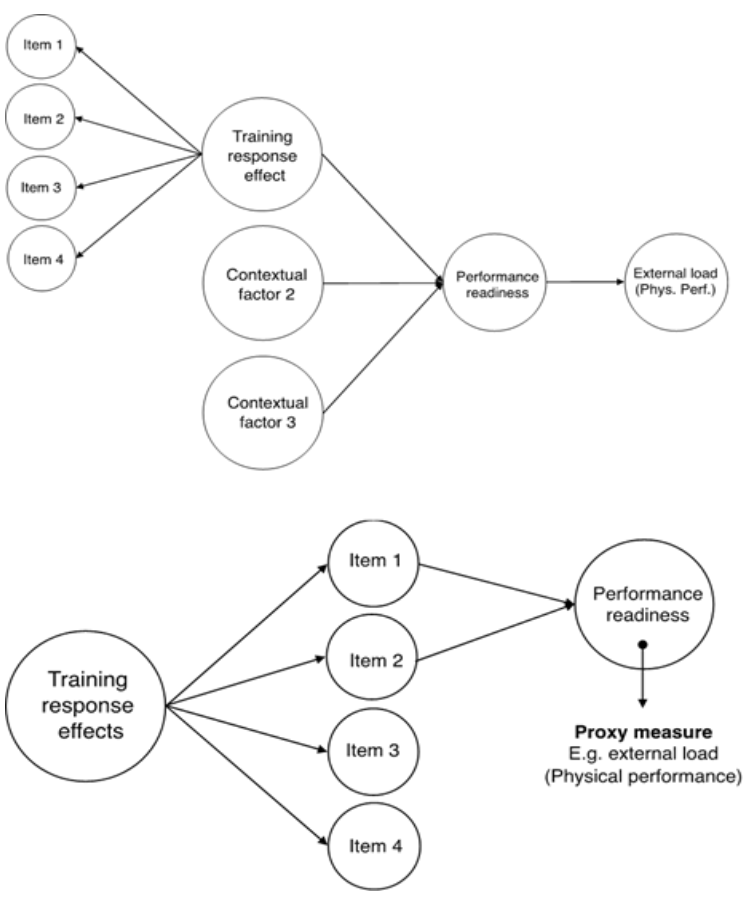

the correlations between physical performance and the single items but in the summary score (assuming the summary score has been validated). In example B, our interest is specifically the single items of which some are not expected to be correlated. In that case, even if the items combined measure a specific training effect construct, our focus is on single items as we are interested in their isolated association to another construct, which is performance readiness. Simply calculating correlations would not be useful without a reference framework, and associations would not be interpretable. Exploratory correlational studies are clearly possible, but at least two issues should be considered. First, the finding can only be used to generate
Figure 2. Example A shows a 4 items reflecting and used to obtain a summary score for a training response effects. This response measure, together with other two measures of contextual factor, are supposed to reflect or determine (depending on the model used) performance readiness. A study may want to see whether training effect measures and contextual factors belongs to the same construct (reflective model): performance readiness. And/or, whether this summary score (combining the three factors) that is supposed to measure performance readiness, is related to physical performance, which is the criterion for the validation.

Example B shows, instead, that 2 out of 4 items measuring training effects are supposed to also reflect performance readiness. A study may test this hypothesis, where item 1 and 2 are hypothesized to be associated (converge), and 3 and 4 don't associate (diverge). If there is not a validated measure of performance readiness, physical performance can be used as proxy measure (surrogate), when performance readiness is, by operational definition, associated to the amount of physical activity completed in the training or competition.

Figure 2. Hypothetical examples of subjective training effects measures

readiness. We developed two hypotheses presented in Figure $2 \mathrm{~A}$ and $\mathrm{B}$.

A) The sum of single item scores are used to measure a training effect that is supposed to reflect performance readiness. Therefore, an association between their summary score, eventually plus measures of contextual factors, and performance is expected.

B) Only two of these items are supposed to measure performance readiness. Convergence of these two items (correlation with physical performance) and divergence for others (no or weaker correlation with physical performance) are expected.

Clearly, depending on the hypothesized relations, the presence or lack of associations provide or do not provide evidence for validity of these items as measures of performance readiness. In example $A$, there is no interest in hypotheses that need to be subsequently tested and are not findings that can suggest how or whether to implement these items in the monitoring process. Second, the multiple associations increase the risk of alpha inflation and this should be considered when interpreting the results and/or building hypotheses based on the findings.

These are hypothetical examples and although based on background knowledge and theories, different kinds of relations and/or causal structures can be hypothesized and tested. However, in validation studies these predictions should be explicitly declared and the conceptual framework (or theory) supporting these hypotheses should be presented. Similarly, the nature and real goal of the study should be explicitly reported (e.g. confirmatory, exploratory), otherwise a fishing expedition in the data lake 
could be generated (adding confusion). Again, we underline it is important to differentiate whether a measure is an attempt to quantify an effect, an individual or contextual, or an effect influencing individual or contextual factors. It is also important to understand how single items, or composite scores, are supposed to influence the associated constructs. This can have a profound effect on the validation process and interpretation of the results. Validating measures is theoretically complex and for this reason necessitates a reference conceptual framework.

\section{Limitations}

Given the qualitative approach of this study, researcher bias is a potential limitation that should be acknowledged. We have assumed, as generally accepted, that optimizing training using measures of the constructs of the training process is superior in terms of sport performance outcomes. However, this assumption is based on existing training theory, experience and inductive reasoning, but not experimental studies. Furthermore, our conceptual framework provides a tentative theory of the phenomena under investigation (physical training), and may need refinement, verification or further explanation.

\section{Summary and Conclusion}

A conceptual framework synthesizes evidence by conceptualizing a phenomenon. Furthermore, it allows for a rigorous, valid and reliable research process, and may serve as a practical tool for interpretation of measures collected in training monitoring. In the development of our conceptual framework, we have built on existing frameworks and models to better explain the training process and its outcomes. In this paper, we have described the conceptual framework development process and presented operational definitions. We introduced and expanded on four concepts: training effects and its possible measures, sport performance outcomes, and individual and contextual factors. Additionally, we explained the relevance and interconnections of these concepts within the training process. The resulting conceptual framework is coherent and fits conceptually available models such as IR-Banister and PerPot. Finally, we presented examples of theoretical (role in the validation process) and practical applications (monitoring, training tolerance, periodization) based on the current conceptual framework. These examples were clearly hypothetical to practically demonstrate how the use of a framework may clarify and explicitly present precise and theoretically grounded testable hypotheses. This provides a useful overarching model for understanding the scientific literature regarding training and additionally guides the development, implementation, and evaluation of a more comprehensive and transparent approach to athlete monitoring and validation of measures.
Acknowledgements: We would like to thank Professor Fabio Nakamura and Carlo Buzzichelii for their assistance.

\section{References}

1. Maxwell, J.A., Conceptual Framework: What Do You Think Is Going On?, in Qualitative research design: An interactive approach (2nd Ed.). 2005, SAGE Publications: Thousand Oaks, CA.

2. Victora, C.G., et al., The role of conceptual frameworks in epidemiological analysis: a hierarchical approach. Int J Epidemiol, 1997. 26(1): p. 224-7.

3.Hernán, M.A., S. Hernández-Díaz, and J.M. Robins, A structural approach to selection bias. Epidemiology, 2004. 15(5): p. 615-25.

4. Greenland, S. and B. Brumback, An overview of relations among causal modelling methods. Int J Epidemiol, 2002. 31(5): p. 10301037.

5. VanderWeele, T.J. and J.M. Robins, Four types of effect modification: a classification based on directed acyclic graphs. Epidemiology, 2007. 18(5): p. 561-8.

6. Cole, S.R., et al., Illustrating bias due to conditioning on a collider. Int J Epidemiol, 2009. 39(2): p. 417-420.

7. Impellizzeri, F. and S. Marcora, Test Validation in Sport Physiology: Lessons Learned from Clinimetrics. Int J Sports Physiol Perform, 2009. 4: p. 269-77.

8. Gimeno-Santos, E., et al., Validity of instruments to measure physical activity may be questionable due to a lack of conceptual frameworks: a systematic review. Health Qual Life Outcomes, 2011. 9(1): p. 86.

9. de Vet, H.C.W., et al., Measurement in Medicine: A Practical Guide. . 2011: Cambridge: Cambridge University Press.

10. Camp, W.G., Formulating and Evaluating Theoretical Frameworks for Career and Technical Education Research. J Vocat Educ Train, 2001. 26(1): p. 27-39.

11. Rothman, M.L., et al., Patient-reported outcomes: conceptual issues. Value Health, 2007. 10 Suppl 2: p. S66-75.

12. Jeffries, A.C., et al., Athlete-Reported Outcome Measures for Monitoring Training Responses: A Systematic Review of Risk of Bias and Measurement Property Quality According to the COSMIN Guidelines. Int J Sports Physiol Perform, 2020: p. 1-13.

13. Wilson, I.B. and P.D. Cleary, Linking clinical variables with healthrelated quality of life. A conceptual model of patient outcomes. J Am Med Assoc, 1995. 273(1): p. 59-65.

14. Smoliga, J.M. and G.S. Zavorsky, Team Logo Predicts Concussion Risk: Lessons in Protecting a Vulnerable Sports Community from Misconceived, but Highly Publicized Epidemiologic Research. Epidemiology, 2017. 28(5): p. 753-757.

15. Impellizzeri, F.M., E. Rampinini, and S.M. Marcora, Physiological assessment of aerobic training in soccer. J Sports Sci, 2005. 23(6): p. 583-92.

16. Impellizzeri, F.M., S.M. Marcora, and A.J. Coutts, Internal and External Training Load: 15 Years On. Int J Sports Physiol Perform, 2019. 14(2): p. 270-273.

17. Viru, A. and M. Viru, Nature of training effects. Exercise and Sport Science, ed. W.E. Garret and D.T. Kirkendall. 2000, Philadelphia: Lippincott Williams and Wilkins.

18. Bhattacherjee, A., Social Science Research: Principles, Methods, and Practices. Textbooks Collection. 3. 2012, South Florida, USA: Global Text Project.

19. Lakens, D., Pandemic researchers - recruit your own best critics. Nature, 2020. 581(7807): p. 121. 
20. Sassi, A., Allenamento e sovrallenamento. 1997, Mllano, Italy: EdiErmes.

21. Matveyev, L., Fundamentals of Sports Training. 1977, Moscow. Russia: Fizkultura i Sport Publ. .

22. Vanrenterghem, J., et al., Training Load Monitoring in Team Sports: A Novel Framework Separating Physiological and Biomechanical Load-Adaptation Pathways. Sports Med, 2017. 47(11): p. 2135-2142.

23. Herold, F., et al., A Discussion on Different Approaches for Prescribing Physical Interventions - Four Roads Lead to Rome, but Which One Should We Choose? J Pers Med, 2020. 10(3).

24. Morton, R.H., J.R. Fitz-Clarke, and E.W. Banister, Modeling human performance in running. J Appl Physiol 1990. 69(3): p. 11717.

25. Calvert, T.W., et al., A Systems Model of the Effects of Training on Physical Performance. IEEE T Syst Man Cy B, 1976. SMC-6(2): p. 94-102.

26. Perl, J., PerPot: A metamodel for simulation of load performance interaction. Eur J Sport Sci, 2001. 1(2): p. 1-13.

27. Perl, J., PerPot - a meta-model and software tool for analysis and optimisation of load-performance-interaction. Int J Perf Anal Spor, 2004. 4(2): p. 61-73.

28. Meeusen, R., et al., Prevention, diagnosis, and treatment of the overtraining syndrome: joint consensus statement of the European College of Sport Science and the American College of Sports Medicine. Med Sci Sports Exerc, 2013. 45(1): p. 186-205.

29. Wilder, R.P., et al., Physical fitness assessment: an update. J Long Term Eff Med Implants, 2006. 16(2): p. 193-204.

30. Medicine, A.A.C.o.S., Acsm's Guidelines for Exercise Testing and Prescription. 8th ed. 2002, Philadelphia, PA, USA: Lippincott Williams \& Wilkins.

31. Edwards, R.H.T., Biochemical basis of fatigue in exercise performance. 1983, Champaign, IL Human Kinetics.

32. Lewis, G. and S. Wessely, The epidemiology of fatigue: more questions than answers. J Epidemiol Commun H, 1992. 46(2): p. 927.

33. Coutts, A.J., S. Crowcroft, and T. Kempton, Developing Athlete Monitoring Systems: Theoretical Basis and Practical Applications, in Sport, Recovery, and Performance: Interdisciplinary Insights, M. Kellmann and J. Beckman, Editors. 2017, Taylor \& Francis.

34. Enoka, R.M. and J. Duchateau, Translating Fatigue to Human Performance. Med Sci Sports Exerc, 2016. 48(11): p. 2228-2238.

35. Morgan, W.P., et al., Psychological monitoring of overtraining and staleness. Br J Sports Med, 1987. 21(3): p. 107-14.

36. Peake, J.M., et al., Muscle damage and inflammation during recovery from exercise. J Appl Physiol (1985), 2017. 122(3): p. 559570.

37. Clarke, D. and P. Skiba, Rationale and resources for teaching the mathematical modeling of athletic training and performance. Adv Physiol Educ, 2013. 37 2: p. 134-52.

38. Endler, S., et al., The PerPot Simulated Anaerobic Threshold - $A$ Comparison to Typical Lactate-based Thresholds. Int J Hum Mov Sports Sci, 2017. 5(1): p. 9-15.

39. Halson, S.L., Monitoring training load to understand fatigue in athletes. Sports Med, 2014. 44 Suppl 2: p. S139-47.

40. McLaren, S.J., et al., The Relationships Between Internal and External Measures of Training Load and Intensity in Team Sports: $A$ Meta-Analysis. Sports Med, 2018. 48(3): p. 641-658.

41. Coffey, V. and J. Hawley, The Molecular Bases of Training Adaptation. Sports Med, 2007. 37: p. 737-63.
42. Thomas, K., et al., Neuromuscular Fatigue and Recovery after Heavy Resistance, Jump, and Sprint Training. Med Sci Sports Exerc, 2018. 50(12): p. 2526-2535.

43. Knuiman, P., M.T.E. Hopman, and M. Mensink, Glycogen availability and skeletal muscle adaptations with endurance and resistance exercise. Nutr Metab, 2015. 12(1): p. 59.

44. Baar, K. and S. McGee, Optimizing training adaptations by manipulating glycogen. Eur J Sport Sci, 2008. 8(2): p. 97-106.

45. Bartlett, J.D., J.A. Hawley, and J.P. Morton, Carbohydrate availability and exercise training adaptation: Too much of a good thing? Eur J Sport Sci, 2015. 15(1): p. 3-12.

46. Fitzpatrick, J.F., et al., Sensitivity and reproducibility of a fatigue response in elite youth football players. Sci Med Footb, 2019. 3(3): p. 214-220.

47. Ade, J.D., et al., Physiological characteristics and acute fatigue associated with position-specific speed endurance soccer drills: production vs maintenance training. Sci Med Footb, 2020: p. 1-12. 48. Sparkes, W., et al., The effect of training order on neuromuscular, endocrine and mood response to small-sided games and resistance training sessions over a 24-h period. J Sci Med Sport, 2020. 23(9): p. 866-871.

49. Clarke, N., et al., Direct and indirect measurement of neuromuscular fatigue in Canadian football players. Appl Physiol Nutr Metab, 2015. 40(5): p. 464-73.

50. Tanner, R.K., C.J. Gore, and A.I.o. Sport, Physiological tests for elite athletes, ed. R.K. Tanner, C.J. Gore, and S. Australian Institute of. 2013, Champaign, IL: Human Kinetics.

51. Behm, D.G., et al., Effectiveness of Traditional Strength vs. Power Training on Muscle Strength, Power and Speed with Youth: A Systematic Review and Meta-Analysis. Front Physiol, 2017. 8: p. 423. 52. Speirs, D.E., et al., Unilateral vs. Bilateral Squat Training for Strength, Sprints, and Agility in Academy Rugby Players. J Strength Cond Res, 2016. 30(2): p. 386-92.

53. Moran, J., et al., Variable long-term developmental trajectories of short sprint speed and jumping height in English Premier League academy soccer players: An applied case study. J Sports Sci, 2020: p. 1-7.

54. Fanchini, M., et al., External Responsiveness of the Yo-Yo IR Test Level 1 in High-level Male Soccer Players. Int J Sports Med, 2015. 36(9): p. 735-41.

55. Jones, A.M. and H. Carter, The effect of endurance training on parameters of aerobic fitness. Sports Med, 2000. 29(6): p. 373-86.

56. Tolfrey, K., et al., Physiological correlates of 2-mile run performance as determined using a novel on-demand treadmill. Appl Physiol Nutr Metab, 2009. 34(4): p. 763-72.

57. Joyner, M.J. and E.F. Coyle, Endurance exercise performance: the physiology of champions. J. Physiol, 2008. 586(1): p. 35-44.

58. Baird, M.F., et al., Creatine-kinase- and exercise-related muscle damage implications for muscle performance and recovery. J Nutr Metab, 2012. 2012: p. 960363.

59. Berriel, G.P., et al., Stress and recovery perception, creatine kinase levels, and performance parameters of male volleyball athletes in a preseason for a championship. Sports Med, 2020. 6(1): p. 26-26.

60. Marin, D.P., et al., Oxidative stress and antioxidant status response of handball athletes: implications for sport training monitoring. Int Immunopharmacol, 2013. 17(2): p. 462-70.

61. Tofari, P.J., J.G. Kemp, and S.J. Cormack, Measuring the response to simulated fixture congestion in soccer. Sci Med Footb, 2020: p. 112. 
62. Coutts, A.J. and S. Cormack, Monitoring the training response High-Performance Training for Sports, ed. I.D.J.D.L. (Eds.). 2014, Champaign, Illinois: Human Kinetics

63. Stanley, J., J.M. Peake, and M. Buchheit, Cardiac parasympathetic reactivation following exercise: implications for training prescription. Sports Med, 2013. 43(12): p. 1259-77.

64. Plews, D.J., et al., Training adaptation and heart rate variability in elite endurance athletes: opening the door to effective monitoring. Sports Med, 2013. 43(9): p. 773-81.

65. Saw, A.E., L.C. Main, and P.B. Gastin, Monitoring the athlete training response: subjective self-reported measures trump commonly used objective measures: a systematic review. Br J Sports Med, 2016. 50(5): p. 281-91.

66. Hooper, S.L., et al., Markers for monitoring overtraining and recovery. Med Sci Sports Exerc, 1995. 27(1): p. 106-12.

67. Hooper, S.L. and L.T. MacKinnon, Monitoring overtraining in athletes. Recommendations. Sports Med, 1995. 20(5): p. 321-327. 68. Schnelle, J.F., et al., Evaluation of two fatigability severity measures in elderly adults. J Am Geriatr Soc, 2012. 60(8): p. 152733.

69. Akenhead, R., J.B. Marques, and D.J. Paul, Accelerometer load: $a$ new way to measure fatigue during repeated sprint training? Sci Med Footb, 2017. 1(2): p. 151-156.

70. Decroix, L., et al., Monitoring Physical and Cognitive Overload During a Training Camp in Professional Female Cyclists. Int J Sports Physiol Perform, 2016. 11(7): p. 933-939.

71. Hurdiel, R., et al., Combined effects of sleep deprivation and strenuous exercise on cognitive performances during The North Face ${ }^{\circledR}$ Ultra Trail du Mont Blanc ${ }^{\circledast}$ (UTMB ${ }^{\circledast}$ ). J Sports Sci, 2015. 33(7): p. 670-674.

72. Lysenko, E.A., et al., Signaling responses to high and moderate load strength exercise in trained muscle. Physiol Rep, 2019. 7(9): $p$. e14100.

73. Coles, E., et al., The influence of contextual factors on healthcare quality improvement initiatives: what works, for whom and in what setting? Protocol for a realist review. Syst Rev, 2017. 6(1): p. 168168.

74. Durand-Bush, N. and J.H. Salmela, The Development and Maintenance of Expert Athletic Performance: Perceptions of World and Olympic Champions. J Appl Sport Psychol, 2002. 14(3): p. 154171.

75. Beck, K.L., et al., Role of nutrition in performance enhancement and postexercise recovery. Open Access J Sports Med, 2015. 6: p. 259-267.

76. Nédélec, M., et al., Sleep Hygiene and Recovery Strategies in Elite Soccer Players. Sports Med, 2015. 45(11): p. 1547-59.

77. Chtourou, H., et al., The effect of ramadan fasting on physical performances, mood state and perceived exertion in young footballers. Asian J Sports Med, 2011. 2(3): p. 177-85.

78. Judge, L.W., et al., The Impact of Competitive Trait Anxiety on Collegiate Powerlifting Performance. J Strength Cond Res, 2016. 30(9): p. 2399-405.

79. Caris, A.V. and R.V.T. Santos, Performance and altitude: Ways that nutrition can help. Nutr, 2019. 60: p. 35-40.

80. Burtscher, M., et al., Preparation for Endurance Competitions at Altitude: Physiological, Psychological, Dietary and Coaching Aspects. A Narrative Review. Front Physiol, 2018. 9: p. 1504-1504.

81. Bühlmayer, L., et al., Effects of Mindfulness Practice on Performance-Relevant Parameters and Performance Outcomes in Sports: A Meta-Analytical Review. Sports Med, 2017. 47(11): p. 2309-2321.
82. Gould, D., N. Damarjian, and C. Greenleaf, Imagery training for peak performance, in Exploring sport and exercise psychology, J.L. Van Raalte and B.W. Brewer, Editors. 2002, American Psychological Association. p. 49-74.

83. Kiely, J., Periodization Paradigms in the 21st Century: EvidenceLed or Tradition-Driven? Int J Sports Physiol Perform, 2012. 7(3): p. 242.

84. Gigerenzer, G. and W. Gaissmaier, Heuristic Decision Making. Annu Rev Psychol, 2011. 62: p. 451-82.

85. Nosek, P., et al., Feedback of GPS training data within professional English soccer: a comparison of decision making and perceptions between coaches, players and performance staff. Sci Med Footb, 2020: p. 1-13.

86. Cheung, K., P. Hume, and L. Maxwell, Delayed onset muscle soreness: Treatment strategies and performance factors. Sports Med, 2003. 33: p. 145-64.

87. AIS, A.I.o.S., Prescription of training load in relation to loading and unloading phases of training (2nd Ed). 2020, Australian Sports Commission: Bruce, ACT, Australia.

88. McGahan, J., et al., Relationship between load and readiness to train in a Gaelic football pre-competition training camp. 2018.

89. McGahan, J., et al., Variation in training load and markers of wellness. J Aust Stength Cond, 2020. 27(3): p. 6-14.

90. Ryan, S., et al., Training monitoring in professional Australian football: theoretical basis and recommendations for coaches and scientists. Sci Med Footb, 2019: p. 1-7.

91. Cullen, B.D., A.L. McCarren, and S. Malone, Ecological validity of self-reported wellness measures to assess pre-training and precompetition preparedness within elite Gaelic football. Sport Sci Health, 2020.

92. Mason, B., et al., The Use of Acute Exercise Interventions as Game Day Priming Strategies to Improve Physical Performance and Athlete Readiness in Team-Sport Athletes: A Systematic Review. Sports Med, 2020. 
Table 1. Operational definitions

\begin{tabular}{|c|c|}
\hline Construct & Operational definition \\
\hline Training load & $\begin{array}{l}\text { Training load refers to a construct reflecting the input variable that is manipulated to elicit a desired training } \\
\text { response in athletes. Within this context, load is a generic term which is qualified by the term training in a } \\
\text { fashion similar to other areas of research that have adopted the term load within a variety of contexts (i.e. } \\
\text { allostatic load, cognitive load, mechanical load etc.). Accordingly, training load does not specifically refer to } \\
\text { the forces experienced, as is typical in physics, or any other physical quantity. Training load, as a generic } \\
\text { construct, accommodates a variety of measures of various nature (spatio-temporal, mechanical, psycho- } \\
\text { physiological etc.) which can be described as being external or internal depending on whether the } \\
\text { measurable aspect in question is internal or external to the athlete. }\end{array}$ \\
\hline
\end{tabular}

External training load

Internal training load

Training effects

Acute effects

Chronic effects

Positive effects

Negative effects

Sport Performance outcomes

Contextual factors

Individual factors
In the context of training load, the term external load, implicitly refers to the external training load undertaken by an athlete. External load has been defined as the physical work prescribed in the training plan. Notably, this does not refer to 'work' in the physics sense (force $x$ distance) but more so in a generic manner. Accordingly, the term external load accommodates quantification and prescription in a variety of manners, enabling the use of a diverse range of measures and metrics. Some common measures of external load include GPS derived units (speed, accelerations, etc.) and level of resistance.

As per external load, the term internal load implicitly refers to internal training loads, where the term training is the qualifier of load. In the context of training load, internal load typically refers to the psycho-physiological stress experienced by an athlete during the exercise or the training session. Therefore, the concept of internal load incorporates all the psycho-physiological responses initiated to cope with the requirements elicited by the external load, i.e. the execution of the exercise (single or sequence) according to the modalities prescribed by the coach. Despite internal loads typically being psycho-physiological in nature (essentially due to relative ease of application and quantification), the stress and strain experienced by specific tissues in response to an applied force is also internal and should therefore also fall within this category.

Effects caused and occurring after the training session, that can be assessed using functional, physiological, subjective, biomechanical and other measures.

Effects induced by one training session to one week of training, that requires brief time (e.g. less than one week) to occur and to return to baseline once training is stopped.

Effects induced by more than one week of training, that requires longer time (e.g. more than one week) to occur and to return to baseline once training is stopped.

Acute or chronic response that directly improves the sport performance outcome

Acute or chronic response that directly impairs the sport performance outcome.

The outcome as a result of the balance between the positive and/or negative training effects.

All the factors not part of the main process (physical training) such as environmental, social, cultural factors, etc that can influence the training process or the training outcome. These factors have an integrated relationship with all components of the conceptual framework, including bidirectionality with training effects.

Characteristics of the individual athlete such as genetics, psychological traits and states, training background, etc. that can influence the training process or the training outcome These factors have an integrated relationship with all components of the conceptual framework, including bidirectionality with training effects. 